\title{
NOISE PREDICTION, CALCULATION AND MAPPING USING SPECIALIZED SOFTWARE
}

\author{
ALINA PETROVICI ${ }^{*}$, CLAUDIA TOMOZEI ${ }^{1}$, RICARDO GEY FLORES ${ }^{2}$, \\ FLORIN NEDEFF ${ }^{1}$, OANA IRIMIA ${ }^{1}$ \\ 1 "Vasile Alecsandri” University of Bacau, Calea Marasesti 157, Bacau, 600115, Romania \\ ${ }^{2}$ Laboratory of Acoustic Engineering, University of Cadiz, CASEM. Av. República Saharaui \\ s/n. 11510 Puerto Real (Cádiz), Spain
}

\begin{abstract}
This paper presents a set of software for prediction, calculation and noise mapping. These are useful both in assessing noise and adopting practical measures in order to reduce noise in areas with intense traffic. The software, through their working tools, allows making comparisons concerning noise before and after implementing noise reduction measures. The analyzed software's of noise prediction, calculation and mapping are specialized for noise generated by traffic.
\end{abstract}

Keywords: traffic noise, noise map, software, prediction

\section{INTRODUCTION}

Road traffic is a serious problem in terms of environmental protection, with the increasing number of vehicles and the continuous development of technology. It is, therefore, necessary to conduct regular assessments to identify urban areas where there are high noise levels that exceed allowable noise limits. These assessments can be performed through on-site measurements or through predictions, using specially designed software. After 20 years of continuous development of the technology, noise mapping software is a professional tool, widely used by many experts from different backgrounds and with great experience in various applications, data and software. The manner of using the software is very important because it can easily influence the quality of the results in the noise mapping process $[1,2]$.

When it comes to properly interpret the noise generated by traffic, data processing software is very useful. Normally, all the software are based either on acoustic measurements or predictions, taking into account in case of traffic noise, various aspects like speed limits, categories of street, asphalt surface (level of absorption of noise). These two ways of analyzing noise can also go together by introducing data about the analyzed area (topographic features, infrastructure, exposed population, etc.) and data concerning the measured noise. The noise map that will be generated after introducing all these parameters will provide later an overview on the analyzed area.

Noise maps are produced by specialized computer software that calculates the noise level in specific areas, showing how noise propagates from the considered sources. This software can also consider elements that affect the dispersion of noise like the buildings, the shape of the land, the capacity of an area to absorb noise (cultivated fields) or to reflect noise (concrete areas, or water surface) [3]. They also take into account the obstacles in the area which can be: barriers, the shape and the acoustical characteristics of the terrain, meteorological conditions, and more [4].

\footnotetext{
${ }^{*}$ Corresponding author, email: am.petrovici@yahoo.ro

(C) 2015 Alma Mater Publishing House
} 


\section{SOFTWARE FOR PREDICTION, CALCULATION AND NOISE MAPPING}

The software for noise prediction, calculation and mapping is specialized for analyzing internal and external noise, differently. This software has different applications for each type of analysis. In this paper, there are analyzed some software with useful applications on assessment, prediction and mapping of the external noise, as the main source of noise targeted is the road traffic. Road traffic noise contribution to the total noise pollution is about $80 \%$, for which regularly assessments are needed on this type of noise.

\subsection{IMMI Software}

IMMI software is useful in estimating the external noise projects in urban areas. It is capable of generating a noise map of an area through importing a cadastral map and by introducing data obtained from measurements of the noise level in the studied area [1]. At the same time, it is capable to work with a model of a digital map which has data about topography, streets, buildings, green areas, transport infrastructure, population distribution. With its help, it can be achieved simultaneous representation of noise levels in the measured points, and also the contour curves of a constant level of the areas corresponding to a constant value of the equivalent noise level [1]. It also allows 3D visualization of noise propagation [5] in a certain area (Figure 1).

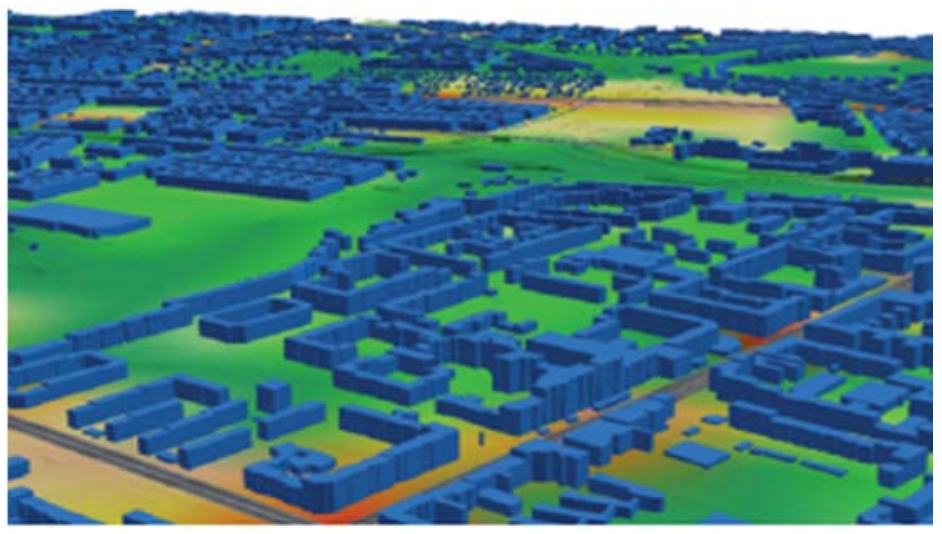

Fig. 1. Noise calculation in 3D view [5].

In order to achieve the noise map, is executed in advance the topographic map of the area (or it is imported the cadastral map of the study area). In this format, it must be marked the point(s) where measurements were made and must be inserted the measured values of the equivalent sound pressure level, $\mathrm{L}_{\text {Aeq. }}$. A noise map is automatically generated, and later the representation of noise dispersion in the studied area is obtained [1].

\subsection{The ambient noise prediction software - CadnaA}

CadnaA is a software used to predict noise level with components used for calculation, presentation, assessment and prediction of the environmental noise. With this software, it can be analyzed the noise generated by an industrial plant, noise from a parking lot, a road or a railway or even the noise from a whole city or an urban area.

The main features of the CadnaA software consist in [6]:

- Comparisons of different planning scenarios;

- Automatically optimization of a noise barrier positioned along a street or railway;

- View noise reduction scenarios;

- Monitoring the noise distribution on all available pathways.

The software CadnaA has been widely used in US urban noise mapping, with the implementation of the European Directive 49/2002 on the assessment and management of environmental noise. This Directive was a catalyst for the development of noise models that show how noise propagates from different sources. In the article Community and Regional Noise Mapping in the United States [7], it is described a noise mapping project in the region of Chittenden, Vermont, where it was used the CadnaA software (Figure 2). Here it was watched how the propagation of noise is affecting the residents in the sensible areas. The purpose of using the software is to find ways of monitoring more efficient the noise in the communities [7]. 


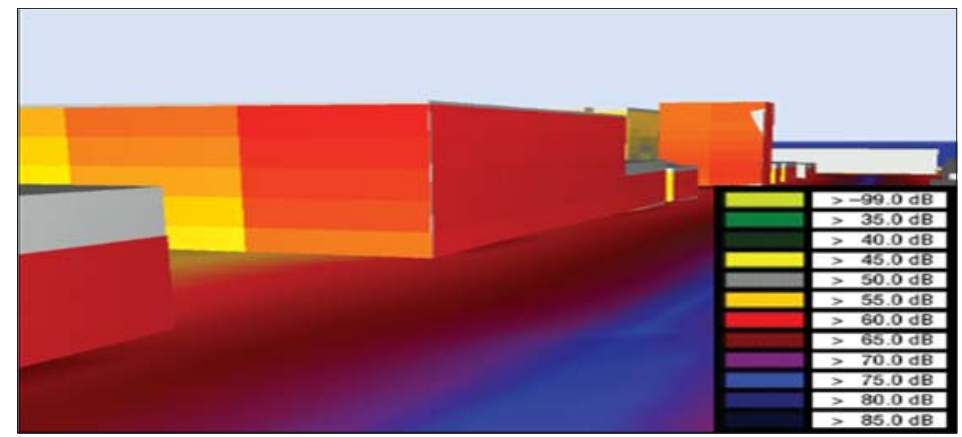

Fig. 2. Noise propagation on a street section of a town from Chittenden VT, 3D view, CadnaA [7].

\subsection{The SPM9613 Community Noise Prediction software}

SPM 9613 is a low-cost computer software used for the analysis of the environmental noise emitted from a variety of sources. The software is based on ISO 9613, 1 and 2. It provides calculations from specific terrain points and predictions of weighted noise level $\mathrm{A}$ and $\mathrm{C}$. The software has a Windows interface and allows multi-source predictions and noise distribution predictions with noise barriers and reflective surfaces [1].

\subsection{CUSTIC software}

With this software, noise pollution analysis is performed by: noise control, environment modeling, and environmental impact assessment. The program calculates the noise level at each point of space for each of the noise sources and under normal air conditions. Through the simulation system of noise dispersion processes, it is quickly and efficiently evaluated the environmental noise pollution from the analyzed area. The program is based on Microsoft Windows operating system [1].

The CUSTIC software can also estimate road traffic noise by introducing the average speed of the vehicles involved in traffic and also by introducing the statistics concerning the number of the vehicles [8].

\subsection{Predictor-LimA Software Suite Type 7810}

Few years ago Lima and Predictor were two different software used separately in different analysis, but in present these two data computer systems merged, in order to provide an accurate tool in any kind of noise project [9].

As there were compared in many studies for example [10], both of these data computer systems have some similar characteristics like considering the following aspects when calculate noise: meteorology variables, sound attenuation of the terrain, incorporate direction of the source, identify the contribution of each source, consider punctual, lineal sources and areas, calculate individual receptors, design of acoustic barriers etc. This software is also integrating very well with other systems like ArcGIS.

Like others noise prediction software, it can be used to evaluate various sources of noise (industry, railroad traffic, road traffic). An important aspect is that it incorporates various standards and noise models which is very useful when calculating noise, because it reduces the time spent to search for this kind of required data.

\subsection{Acoustic Determinator 7816 software}

Acoustic software is a tool for determining sound power emission of industrial noise sources by measuring sound pressure levels in the environment. Using this software it can be determined the sound power level, $\mathrm{L}_{\mathrm{w}}$, to be used in environmental noise calculation software; data post-processing through mediation, addition, or arithmetic subtraction [1].

To read the measurement data, the software Acoustic Determinator uses general and standard parameters in order to define the measurement properties. The general parameters can include: source description, measurement time, sound type, temperature, wind speed, humidity. The standard parameters can include: multiple measuring surfaces, multiple measuring points, sound pressure level, and corrections, background noise [11].

The software is helpful in determining the noise level from different external sources, using field measurements made in accordance with numerous national and international standards [12]. 


\subsection{MITHRA SIG Software}

MITHRA is intended to design noise maps (Figure 3) for cities, or certain regions of the cities [13]. MITHRASIG software was used in comparative studies with other noise mapping software, to evaluate noise levels in different regions and to highlight urban population exposure both in urban and rural areas, on various noise sources (road traffic, railway etc.). To analyze these noise sources, the following indicators are used: $\mathrm{L}_{\mathrm{den}}, \mathrm{L}_{\mathrm{dn}}, \mathrm{L}_{\mathrm{night}}, \mathrm{L}_{\text {evening, }}$ $\mathrm{L}_{\mathrm{day}}[14]$.

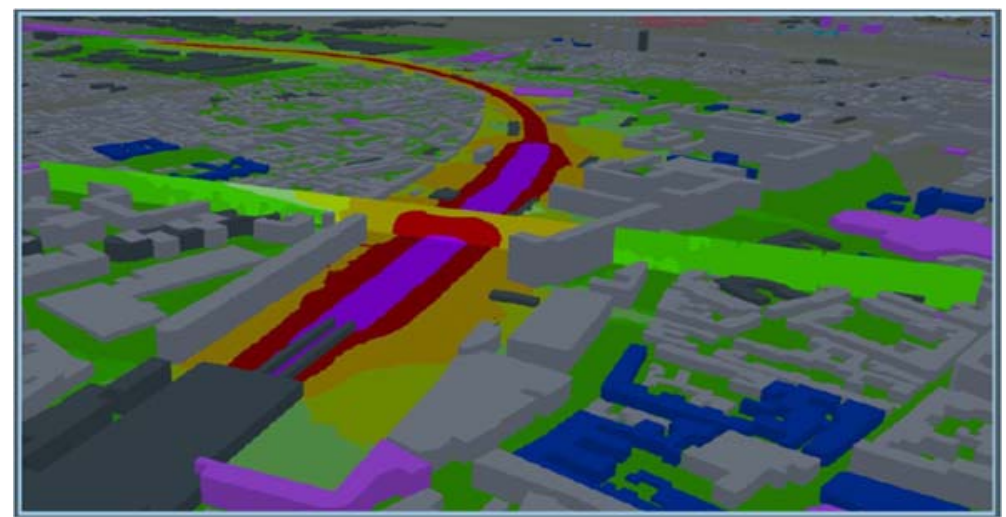

Fig. 3. Noise map generated by MITHRA SIG [10].

The applications of this software are:

- It simulates the propagation of noise from certain sources: road, rail etc.;

- It performs dynamic noise maps: maps to the receiver placed by the operator, 2D maps, 3D maps showing the distribution of noise on building facades;

- It displays information about the results of the prediction and noise measurements;

- It analyzes data by generating noise maps before and after implementing the measures to reduce noise, (eg. the implementation of noise barriers, or forwarding traffic);

- It calculates the population exposed to noise;

\subsection{The SoundPLAN software}

SoundPLAN is a noise modeling software used since 1986. The main applications of this software consist in prediction, assessment, and mapping of environmental noise [15]. This software is useful in projects of construction and planning of new roads because it presents characteristics concerning the optimization of noise barriers (Figure 4), with tools used to analyze the relationship between costs and benefits. In areas where noise barriers are necessary, the instruments of the software show the area of minimizing the size of a protective barrier, thus, substantially reducing construction costs [16].

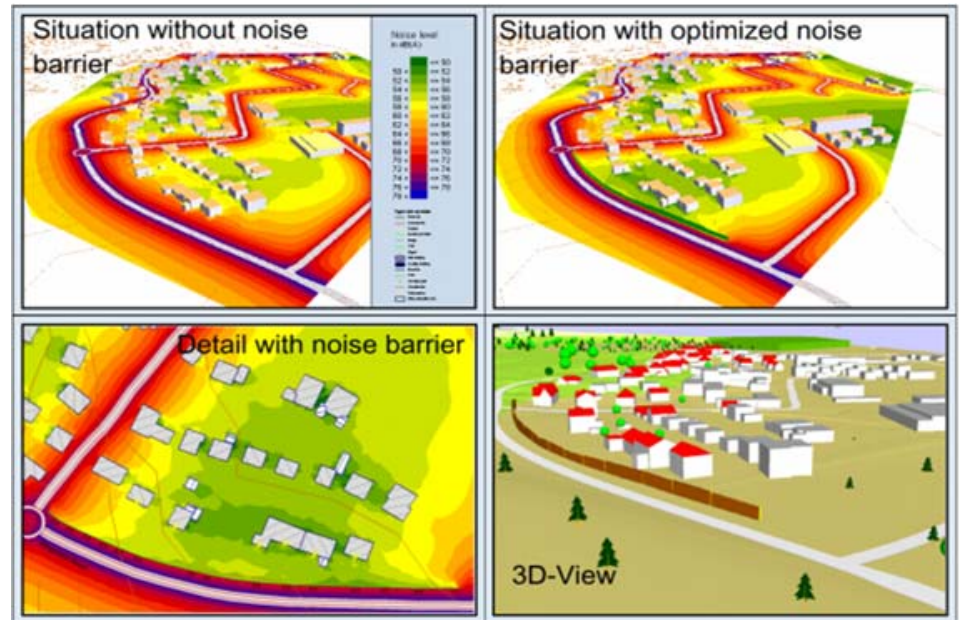

Fig. 4. Projections concerning the optimization of noise barriers generated by SoundPLAN [16].

The specialized literature [17] presents the results of a study on the influence of urban characteristics on the ambient noise in the city of Aracaju (Brazil). The study, which involved making field measurements and acoustic 
simulations using the software SoundPLAN, began with an analysis of the current scenario, followed by the creation and simulation of hypothetical scenarios in areas yet unoccupied in the studied regions. The input data in this study included: sound power of noise sources; data traffic: traffic flow, the percentage of heavy vehicles, street pavement to calculate noise from roads, streets; geometry area (topography), the location and physical characteristics of buildings, enclosure walls (barriers) and other design elements that can interfere in the propagation of the exterior noise. The study results showed that the physical characteristics of the urban areas such as building density, the existence of free spaces and the buildings shape and position, have significant influence on the environmental noise [17].

The advantages of using this software are the following [16]:

- It performs assessment for small construction projects, and it can be used for noise mapping in large cities;

- It performs noise assessment for certain areas before placing a road;

- It makes comparisons between different scenarios concerning the level of noise generated before and after the implementation of noise reduction measures;

- It highlights the areas with high noise levels;

- It performs calculations on the effectiveness of noise barriers.

\section{DISCUTIONS}

Road traffic is one of the main sources of external noise, analyzed in an urban area with this kind of software. For an accurate assessment of traffic noise, there are necessary a set of data. The input data used in the calculation and mapping of road traffic noise concerns: infrastructure and topographical features of the analyzed area, the influence of the acoustical reflections caused by the existence of the buildings on the opposite side of the road, vehicles velocity, number of vehicles involved in traffic etc.

The evaluation of traffic noise through noise mapping is a macroscopic evaluation, so it does not present the situation in the area in a very detailed manner. Later, before identifying the "hot spots", a microscopic analysis can be performed.

The software presented in this paper have as common feature, the assessment for external noise. The assessment is based both on noise measurements, noise estimations or combinations between these two aspects.

The noise maps generated by this software show an overview of the analyzed area from the acoustical point of view. They highlight the areas where the noise levels exceed the maximum allowable limits, and they are used for different type of construction projects like roads or residential areas.

With this software can be achieved comparative studies on areas where noise is a problem. Thus, they can provide data on the noise level after implementing some corrective actions (e.g. installing noise barriers).

Another important feature of the software is to provide simulations and animations showing clearly how noise propagates from a mobile noise source (SoundPLAN, MITHRA-SIG, CadnaA etc.).

A very practical aspect of this software is related to the fact that they incorporate elements from different international standards and standardized methods for assessing noise (NMPB Routes, CoRTN, RLS etc.).

\section{CONCLUSIONS}

The prediction and noise mapping using the software is a useful tool to analyze the current situation of external noise. With this software is shown comprehensive information on the dispersion of noise in a given urban area or even on an entire city. Thus, can be achieved an efficient monitoring in areas where there are high noise levels. In most cases, at a location, noise can be combination of local sources such as a combination of noise from road and rail.

There are some technical and practical reasons why the noise maps are generated mostly by using predictions/simulations instead of using noise measurements. Thus, the development of noise maps based on measurements requires a large number of measurements which also requires long periods of time and highly costs. 


\section{REFERENCES}

[1] Tomozei, C., Panainte, M., Lazar, G., Nedeff, F., Nedeff, V., Reducerea poluării fonice în mediul industrial, Alma Mater Bacau, 2014, p. 132-137.

[2] Douglas, M., Van Banda, E.H., Good practice in the use of noise mapping software, Applied Acoustics, vol. 72, 2011 p. 527-533.

[3] CNADNR, Hărțile de zgomot, online at: http://213.177.10.50:5555/Intrebari.htm (2.10.2014).

[4] Harta de zgomot, 2014, online at:http://ro.wikipedia.org/wiki/Hart\%C4\%83 de_zgomot.

[5] Noise mapping and noise prediction with IMMI brochure online at: http://download.woelfel.de/fileadmin/ftp/Downloads/IMMI_Downloads/Datenblaetter_Englisch/IMMI_product_ information.pdf).

[6] CadnaA Software brochure online at: http://www.datakustik.com/fileadmin/user_upload/PDF/2012/CadnaA_at_a_glance_english.pdf).

[7] Kaliski, K., Duncan, E., Cowan, J., Community and Regional Noise Mapping in the United States, Sound and Vibration, 2007, p. 14-17.

[8] Hadzi-Nikolova M., Mirakovski D., Delipetrov T., Arsov P., Noise Dispersion Modelling in Small Urban Areas with CUSTIC 3.2 Software, International Journal of Emerging Technology and Advanced Engineering, Vol. 2, 2012, p. 84-88.

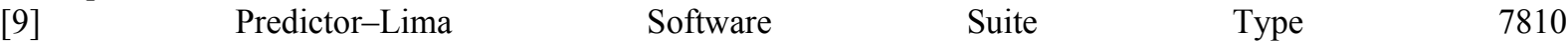
http://www.bksv.com/Products/EnvironmentManagementSolutions/Noise\%20Mapping\%20and\%20Prediction/pr edictor-lima-7810.

[10] Martínez, E.; Recuero, M., Comparison of Computer Software for External Noise Prediction, 19th International Congress on Acoustics Madrid, 2-7 September 2007.

[11] Acoustic Determinator Type 7816, Product Data on line at: https://www.softnoise.com/pdf/bp2003.pdf 12.01.2015.

[12] Acoustic $\quad$ Determinator $\quad$ Type 7816 http://www.bksv.com/Products/EnvironmentManagementSolutions/Noise\%20Mapping\%20and\%20Prediction/a coustic-determinator-7816.

[13] MITHRA SIG - Acoustic Mapping Software Brochure on line at: http://geomod.fr/gmd-societe/gt-accueil/gtlogiciels/gt-log-mithra-suite/gt-log-mithra-sig/.

[14] Lercher, P., De Greve, B., Botteldooren, D., Baulac, M., Defrance, J., Rüdisser, J., The effect on annoyance estimation of noise modeling procedures, 9th International Congress on Noise as a Public Health Problem (ICBEN), Foxwoods, CT, 2008.

[15] SoundPLAN Acoustics presentations online at: http://www.soundplan.eu/english/soundplan-acoustics/.

[16] SounPLAN, Noise control walls and planning, online at:http://www.acoustics.gr/SoundPlan.pdf.

[17] Italo, C., Guedes, M., Bertoli, S.R., Zannin, P.H.T., Influence of urban shapes on environmental noise: A case study in Aracaju — Brazil, Science of the Total Environment 412-413, 2011, p. 66-76. 